\title{
Kaempferol Modulates DNA Methylation and Downregulates DNMT3B in Bladder Cancer
}

\author{
Wei Qiu $\quad$ Jun Lin ${ }^{a} \quad$ Yichen Zhu ${ }^{a}$ Jian Zhang ${ }^{a}$ Liping Zeng ${ }^{b, c}$ Ming Su ${ }^{d}$ Ye Tian ${ }^{a}$ \\ ${ }^{a}$ Department of Urology, Beijing Friendship Hospital, Capital Medical University, Beijing, ${ }^{\text {bThe Clinical }}$ \\ Laboratory of No.261 Hospital of the People's Liberation Army, Beijing, 'The State Key Laboratory of \\ Medical Genetics, Central South University, Changsha, 'State Key Laboratory of Cardiovascular Disease, \\ Fuwai Hospital, National Center for Cardiovascular Disease, Chinese Academy of Medical Sciences and \\ Peking Union Medical College, Beijing, China
}

\section{Key Words}

Bladder cancer • Kaempferol • DNA methylation • DNMT3B

\begin{abstract}
Background: Genomic DNA methylation plays an important role in both the occurrence and development of bladder cancer. Kaempferol (Kae), a natural flavonoid that is present in many fruits and vegetables, exhibits potent anti-cancer effects in bladder cancer. Similar to other flavonoids, Kae possesses a flavan nucleus in its structure. This structure was reported to inhibit DNA methylation by suppressing DNA methyltransferases (DNMTs). However, whether Kae can inhibit DNA methylation remains unclear. Methods: Nude mice bearing bladder cancer were treated with Kae for 31 days. The genomic DNA was extracted from xenografts and the methylation changes was determined using an Illumina Infinium HumanMethylation 450 BeadChip Array. The ubiquitination was detected using immuno-precipitation assay. Results: Our data indicated that Kae modulated DNA methylation in bladder cancer, inducing 103 differential DNA methylation positions (dDMPs) associated with genes (50 hyper-methylated and 53 hypo-methylated). DNA methylation is mostly relied on the levels of DNMTs. We observed that Kae specifically inhibited the protein levels of DNMT3B without altering the expression of DNMT1 or DNMT3A. However, Kae did not downregulate the transcription of DNMT3B. Interestingly, we observed that Kae induced a premature degradation of DNMT3B by inhibiting protein synthesis with cycloheximide (CHX). By blocking proteasome with MG132, we observed that Kae induced an increased ubiquitination of DNMT3B. These results suggested that Kae could induce the degradation of DNMT3B through ubiquitin-proteasome pathway. Conclusion: Our data indicated that Kae is a novel DNMT3B inhibitor, which may promote the degradation of DNMT3B in bladder cancer.

W. Qiu and J. Lin contributed equally to this work.

Ye Tian

KARGER
Department of Urology, Friendship Hospital Affiliated to Capital Medical University, 95th Yong An Road, Xi Cheng District, Beijing, 100050 (China)

E-Mail youyiminiao@126.com 


\section{Introduction}

Bladder cancer is a common urological malignant tumor that severely affects patients' quality of life. Similar to most other cancers, the pathogenesis of bladder cancer remains poorly understood. Accumulated evidences indicate that the methylation modification of genomic DNA is closely associated with bladder cancer [1-5]. The hyper-methylation of tumor-suppressive genes and the hypo-methylation of oncogenes regulate the proliferation and metastasis of bladder cancer [4-6]. Therefore, the re-activation of tumor suppressors and/or the inhibition of oncogenes by modulating DNA methylation with effective and lowtoxicity reagents may be a strategy for the prevention and treatment of cancer $[7,8]$.

Kaempferol (Kae) is a natural flavonoid that occurs widely in many fruits and vegetables. Kae was reported to be an anti-tumor reagent against many cancers, such as breast cancer, pancreatic cancer, prostate cancer, and lung cancer [9-12]. Previously, we found that Kae could suppress bladder cancer by inducing apoptosis both in vitro and in vivo [13,14]. These data highlight that this compound may be a new candidate for the prevention and treatment of bladder cancer.

Many flavonoids are potent anti-tumor chemicals. The well-known flavonoid (-)-epigallocatechin-3-0-gallate (EGCG) strongly inhibits DNA methylation by interfering with DNA methyltransferases (DNMTs) [15]. All of the flavonoids, including EGCG and Kae, share a unique flavan nucleus in their structures [15]. This nucleus structure is responsible for its DNA methylation inhibitory activity [16]. Due to the inhibitory effects of Kae on bladder cancer and its chemical structure, we hypothesized that Kae might suppress the DNMTs and regulate DNA methylation in bladder cancer. To investigate these effects, we constructed bladder cancer T24 cell-derived xenografts models and treated with Kae. We found Kae induced the decrease of DNMT3B and modulated DNA methylation in bladder cancer.

\section{Materials and Methods}

Main chemicals and reagents

Kae was purchased from Sigma-Aldrich (St. Louis, MO, USA). For in vitro studies, Kae was dissolved in DMSO (Sigma-Aldrich) to produce a stock solution of $80 \mathrm{mM}$ and was stored at $-20^{\circ} \mathrm{C}$; a final concentration at $40 \mu \mathrm{M}$ was used. For in vivo studies, Kae was dissolved in sterile corn oil (Sigma-Aldrich). Fetal bovine serum (FBS), phosphate-buffered saline (PBS), TRIzol reagent and RPMI 1640 medium were purchased from Thermo Fisher Scientific (Schuylerville, NY, USA). Rabbit anti-5-methylcytosine, DNMT1, DNMT3A, DNMT3B, GAPDH primary antibodies and goat anti-rabbit, goat anti-mouse secondary antibodies were all obtained from Cell Signaling Technology (Beverly, MA, USA). A mouse anti-ubiquitin (Ub) antibody was purchased from Santa Cruz (Dallas, TX, USA).

\section{Cell culture}

The T24 and 5637 bladder cancer cell lines, two widely used cell lines in bladder cancer research, were cultured in RPMI 1640 medium containing 10\% FBS. The cultures were incubated at $37^{\circ} \mathrm{C}$ in a humidified atmosphere containing $5 \% \mathrm{CO}_{2}$.

\section{Animal experiments}

The animal experiments were approved by the Beijing Friendship Hospital Animal Care and Use Committee. Bladder cancer xenografts were prepared as previously described [17]. In brief, $3 \times 10^{6} \mathrm{~T} 24$ cells per $100 \mu \mathrm{l}$ cell suspension was injected into the right flank of 5 -week-old BALB/c nude mice. Five days after cell transplantation, the mice were randomly distributed into vehicle and Kae treatment groups (n=5 per group). Kae was administered by an intraperitoneal injection at the dose of $150 \mathrm{mg} / \mathrm{kg}$ every day. The mice in the vehicle group were intraperitoneally injected with an equal volume of corn oil. The tumor size was measured with a vernier caliper. Tumor volume was calculated as (length $\times$ width $^{2}$ )/2. All of the mice were treated for 31 days and then sacrificed for further study. 


\section{Cellular Physiology Cell Physiol Biochem 2017;41:1325-1335

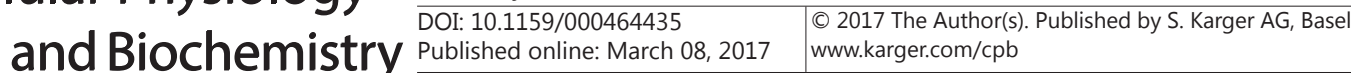 \\ Qiu et al.: Kaempferol Modulates DNA Methylation in Bladder Cancer}

\section{DNA extraction}

The genomic DNA of tumor tissues was extracted using a TaKaRa MiniBEST Universal Genomic DNA Extraction Kit (Dalian, China) according to the manufacturer's instructions. In brief, the samples were digested with lysis buffer containing Proteinase $\mathrm{K}$ and RNase A at $56{ }^{\circ} \mathrm{C}$. Then, the genomic DNA was separated and purified using a spin column. The concentration was measured using a NanoDrop Lite Spectrophotometer (Thermo Fisher Scientific), and the quality of each sample was evaluated by using an agarose gel electrophoresis assay for the following analysis.

Dot blotting for the detection of global content of 5-methylcytosine

To analyze global DNA methylation, DNA Dot blotting was performed to detect 5-methylcytosine. The protocols were followed according to the manufacturer's instructions provided by Cell Signaling Technology. In brief, the genomic DNA was diluted with nuclease-free water into a range of concentrations, denatured by heating at $95{ }^{\circ} \mathrm{C}$ for $10 \mathrm{~min}$ and immediately chilled on ice for $5 \mathrm{~min}$. Then, the DNA was applied onto nylon membrane and ultraviolet (UV) cross-linked at $1200 \mathrm{~J} / \mathrm{m}^{2}$. The membrane was then blocked with $5 \%$ nonfat milk in Tris Buffered Saline with Tween 20 (TBST) solution for $1 \mathrm{~h}$ at room temperature and incubated in rabbit-anti-5-methylcytosine antibody (1:1000; Cell Signaling Technology) at $4{ }^{\circ} \mathrm{C}$ overnight. Then, the membrane was washed 3 times with TBST and incubated with goat-anti-rabbit secondary antibody at room temperature for $1 \mathrm{~h}$. After that, the membrane was washed 3 times with TBST and the dots were detected using SuperSignal West Femto Maximum Sensitivity Substrate (Pierce, Rockford, IL, USA). The membrane was then washed with nuclease-free water 3 times and stained with methylene blue solution as an internal control for each group.

High-density DNA methylation assay

The DNA methylation profiles were determined at Sangon Biotech Shanghai Co Ltd (Shanghai, China) using Illumina Infinium HumanMethylation 450 BeadChip Arrays (Illumina HM450K V1.2, San Diego, CA, USA), and the protocol was followed according to the manufacturer's instruction. DNA samples from xenografts (3 per group) were bisulfite-converted using a Zymo EZ DNA Methylation kit (Zymo Research, Orange, CA, USA), all of the samples were randomly distributed across the microarray. The Illumina Genomestudio software (San Diego, CA, USA) was used to process the DNA methylation data, and data processing was performed with the IMA package in $\mathrm{R}$ (Illumina). The $\beta$ values were used to represent the methylation levels of individual sites. A total of 592 unreliable loci according to $\mathrm{P}$ values and 1215 loci containing missing $\beta$ values were excluded, leaving 483,770 loci. This microarray covers $99 \%$ of the RefSeq genes with multiple sites in the promoter, including 1500 and 200 base pair (bp) upstream of the transcription start site (TSS), 5'-untranslated regions (UTR), gene body and the 3'-UTR; 96\% of CpG islands with sites in the islands, shores and shelves.

\section{Pyrosequencing methylation analysis}

The methylation status of the CpG rich region (CGG CGG GCA CTC ACA TCA TCT CTG CCA TCA CCG CCG CCG CCG ATG CCC) of WRN (Werner syndrome RecQ like helicase) promoter indicated by the "HM450K" data was determined with pyrosequencing methylation assay. The genomic DNA (1 $\mu \mathrm{g})$ was treated with sodium bisulfite using the EZ DNA Methylation-Gold Kit (Zymo Research) according to the manufacturer's instructions. Then the products of bisulfite-treated genomic DNA were amplified using the following primers: forward primer (FP, biotinylated): 5'-GTT GTA GTA GAG GGG TAG AGA GTT-3'; reverse primer (RF): 5 '- CTC TAC CCT CTC TCC CCT CAT CCC T-3'. The amplifying condition was: $95{ }^{\circ} \mathrm{C}$ for $3 \mathrm{~min}$, $\left[94^{\circ} \mathrm{C}\right.$ for $25 \mathrm{~s}$, $58{ }^{\circ} \mathrm{C}$ for $25 \mathrm{~s}, 72^{\circ} \mathrm{C}$ for $25 \mathrm{~s}$ ] 35 cycles, $72{ }^{\circ} \mathrm{C}$ for $5 \mathrm{~min}$. Pyrosequencing was performed using the following primer: 5'-ACC CCA AAT CTC CAA C-3'. The methylation index was indicated using the average methylation $\%$ of the CpGs.

\section{Protein expression levels}

Whole protein in cells or tissue was extracted using RIPA lysis buffer. The protein $(20-40 \mu \mathrm{g})$ from each group was loaded onto SDS polyacrylamide gels for electrophoretic separation and blotted onto nitrocellulose membranes. Then, the membranes were blocked with 5\% non-fat milk in TBST solution for $1 \mathrm{~h}$ at room temperature. The membranes were soaked in diluted primary antibodies (1:1000 with TBST) at $4^{\circ} \mathrm{C}$ overnight. The membranes were then washed 4 times with TBST and incubated in diluted secondary 


\section{Cellular Physiology Cell Physiol Biochem 2017;41:1325-1335

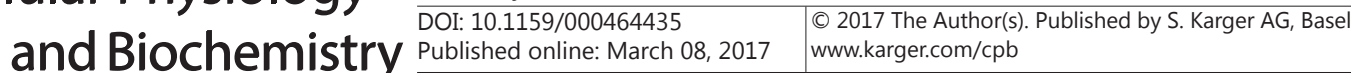 \\ Qiu et al.: Kaempferol Modulates DNA Methylation in Bladder Cancer}

antibody (1:2000 with TBST) for $1 \mathrm{~h}$ at room temperature. Then, the membranes were washed 4 times with TBST solution, and the bands were detected using SuperSignal West Femto Maximum Sensitivity Substrate (Pierce, Rockford, IL, USA). The intensity of each band was scanned with Quantity One software (Bio-Rad, Hercules, CA, USA). The relative intensity of each band was normalized with the corresponding intensity of GAPDH.

Determination of gene transcription levels

Total RNA was extracted using TRIzol reagent, and 500 ng RNA from each sample was used for the reverse transcription using a cDNA synthesis kit (TaKaRa, Dalian, China). The reverse transcription products were diluted with nuclearase-free water to a final concentration at $5 \mathrm{ng} / \mu \mathrm{l}$. For real-time qPCR, 15 ng of cDNA in each reaction was analyzed using the 7500 Real-Time PCR System (Applied Biosystems). The total volume of each reaction was $20 \mu \mathrm{l}$, containing $3 \mu \mathrm{l}$ of diluted cDNA solution, $10 \mu \mathrm{l}$ of $2 \times$ qPCR SYBR $^{\circledR}$ Fast qPCR Mix (TaKaRa), $0.4 \mu \mathrm{l}$ forward and reverse primers (10 pmol/ $\mu \mathrm{l}), 0.4 \mu \mathrm{l} 50 \times$ ROX reference Dye II and $5.8 \mu \mathrm{l}$ nuclearase-free water. GAPDH was employed as an internal control. The relative expression was calculated with the $\triangle \Delta \mathrm{Ct}$ (cycle threshold) method. The following primers were listed: DNMT1 FP: 5'-TAC CTG GAC GAC CCT GAC CTC-3', RP: 5'-CGT TGG CAT CAA AGA TGG ACA-3'; DNMT3A FP: 5'-TAT TGA TGA GCG CAC AAG AGA GC-3', RP: 5'-GGG TGT TCC AGG GTA ACA TTG AG-3'; DNMT3B FP: 5'-GGC AAG TTC TCC GAG GTC TCT G-3', RP: 5'-TGG TAC ATG GCT TTT CGA TAG GA-3'; WRN FP: 5'-TGC AGC CAT TTC TTG TCA AA-3', RP: 5'-GAA GGA CAG TAG ATG ATT GTT GGA-3'; GAPDH FP: 5'-GCC ACA TCG CTC AGA CAC-3', RP: 5'-GCC CAA TAC GAC CAA ATC C-3'. The primer sequences for WRN and GAPDH were used as previously reported [18]. The condition for amplification was: $95^{\circ} \mathrm{C}$ for $5 \mathrm{~min},\left[95^{\circ} \mathrm{C}\right.$ for $25 \mathrm{~s}, 60^{\circ} \mathrm{C}$ for $\left.60 \mathrm{~s}\right] 40$ cycles.

\section{Ubiquitination study}

The cells were treated with or without Kae in the presence of cycloheximide (CHX) (Sigma-Aldrich) at $50 \mu \mathrm{g} / \mathrm{ml}$ to inhibit protein synthesis for 6,12 and $24 \mathrm{~h}$, respectively. The degradation of DNMT3B was detected with western blotting.

The cells were treated with MG132 (Sigma-Aldrich) at a final concentration of $5 \mu \mathrm{M}$ with or without Kae for $6 \mathrm{~h}$. Then, the total protein in each group was extracted and immuno-precipitated with Protein G Magnetic Beads (Thermo Fisher) and rabbit-anti-DNMT3B antibody. The precipitates were washed and eluted. The status of ubiquitination was detected by using regular western blot with an Ub antibody (Santa Cruz). DNMT3B in the total protein of each group were employed as inputs.

\section{Statistical analysis}

The data were indicated as the mean \pm S.D. obtained from at least three independent experiments. The differences between groups were calculated using a two-tailed Student's t-test performed using the IBM SPSS 19.0 software (SPSS Inc., Chicago, IL, USA). The statistical significance was defined as $\mathrm{P}<0.05$. For the differential DNA methylation, the IMA package in R (Illumina) was used. Differential methylation values such as $\Delta \beta$ and $P$ values were calculated. Sites for which $|\Delta \beta| \geq 0.1$ and $\mathrm{P}<0.05$ were considered differentially methylated.

\section{Results}

Kaempferol inhibits bladder cancer growth in vivo

To analyze the effects of Kae on bladder cancer in vivo, bladder cancer xenografts were prepared with T24 cells. Nude mice bearing tumor xenografts were treated with Kae (150 $\mathrm{mg} / \mathrm{kg} /$ day) or vehicle for 31 days. As expected, Kae treatment significantly inhibited the growth of xenografts (Fig. 1A). The volume of the xenografts was smaller in Kae-treated mice than in vehicle-treated mice (Fig. 1B).

Kaempferol does not vary the global content of 5-methylcytosine in bladder cancer

To analyze whether Kae regulates DNA methylation in bladder cancer, total DNA from bladder cancer xenografts was extracted. We firstly evaluated global DNA methylation by 


\section{Cellular Physiology Cell Physiol Biochem 2017;41:1325-1335 and Biochemistry Published onIme: Varch 08, $2017 \quad \begin{aligned} & \text { DOI: 10.1159/000464435 } 2017 \text { The Author(s). Published by S. Karger AG, Basel } \\ & \text { www.karger.com/cpb }\end{aligned}$

Fig. 1. Kaempferol inhibits bladder tumor growth in nude mice bearing T24 cells. Mice were subcutaneously inoculated with $3 \times 10^{6}$ T24 cells and were randomly divided into two groups. Kaempferol (Kae) dissolved in corn oil was injected intraperitoneally every day. The dose of Kae was $150 \mathrm{mg} / \mathrm{kg}$. Control mice were injected with an equal volume of corn oil (vehicle). The tumor growth curve (A) and the appearance of xenografts (B) are shown (scale bar: $2 \mathrm{~cm}$ ). The results are presented as the mean $\pm \mathrm{SD} ;{ }^{*} \mathrm{P}<0.05$ and ${ }^{* *} \mathrm{P}<0.01$ compared with the control group.

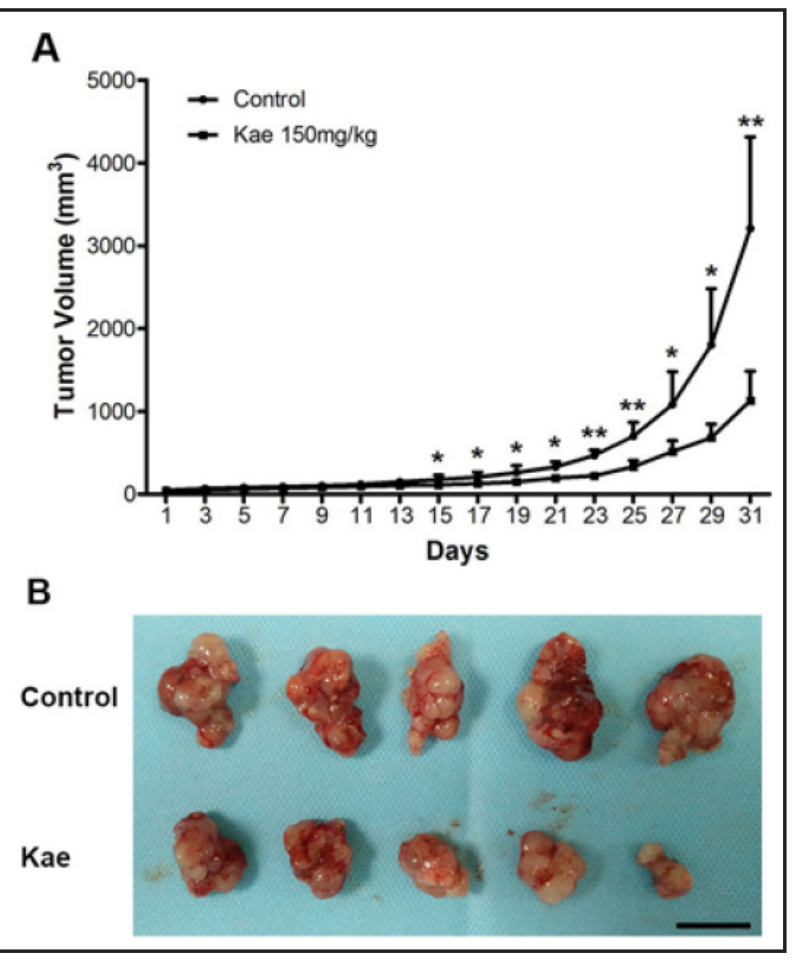

detecting 5-methylcytosine. However, we found that Kae did not alter the global content of 5-methylcytosine in the xenografts (Fig. 2A).

Kaempferol modulates DNA methylation in bladder cancer

To more precisely describe the effects of Kae on DNA methylation, an HM450K microarray was used to detect differential DNA methylation positions (dDMPs). We found that there were 103 dDMPs associated with genes, including 50 hyper-methylated (48.5\%) and 53 hypo-methylated (51.5\%). The dDMPs were associated with 82 genes, and encoded 126 transcripts, including 61 hyper-methylated (48.4\%) and 65 hypo-methylated (51.6\%) (Fig. 2B).

The dDMPs were distributed as follows: $17 \%$ in the island region, $11 \%$ in the N-shelf, $14 \%$ in the $\mathrm{N}$-shore, $6 \%$ in the S-shelf and $11 \%$ in the S-shore. The distribution of dDMPs in the different regions was calculated. We found that the number of hypo-methylated sites in the $\mathrm{CpG}$ island region was larger than that of hyper-methylated sites. These dDMPs distributed in the genes were listed as follows: $35 \%$ in the gene body region, $2 \%$ in the first exon, $8 \%$ in the TSS $200,21 \%$ in the TSS1500, 11\% in the 5'UTR and 3\% in the $3^{\prime}$ UTR. The dDMPs in the TSS1500, TSS200 and 5'UTR regions were more frequently hypo-methylated, whereas in the gene body region, they were more frequently hyper-methylated after Kae treatment.

The differentially DNA methylated regions (dDMRs) were analyzed. We found that 11 dDMRs were associated with coding genes, including 3 hyper-methylated and 8 hypomethylated. In our HM450K microarray data, we found that the TSS200 region of WRN promoter was hypo-methylated. The promoter of this gene contains $\mathrm{CpG}$ rich region that can be regulated by DNMT3B [19]. To validate the DNA methylation, we detected the DNA methylation changes in the TSS200 region of WRN promoter using pyrosequencing methylation analysis. We found that the methylation index was significantly downregulated by Kae in bladder cancer xenografts (Fig. 2C). Consistently, the expression of WRN was increased to 2.0-fold (Fig. 2D). 
A

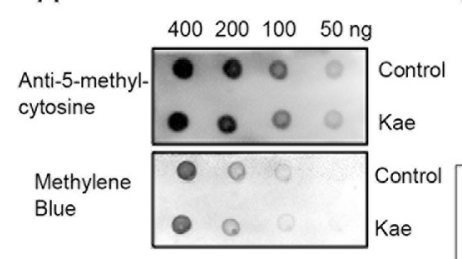

D

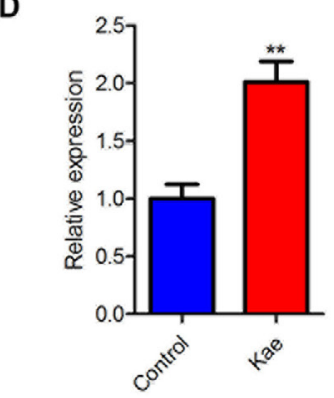

C
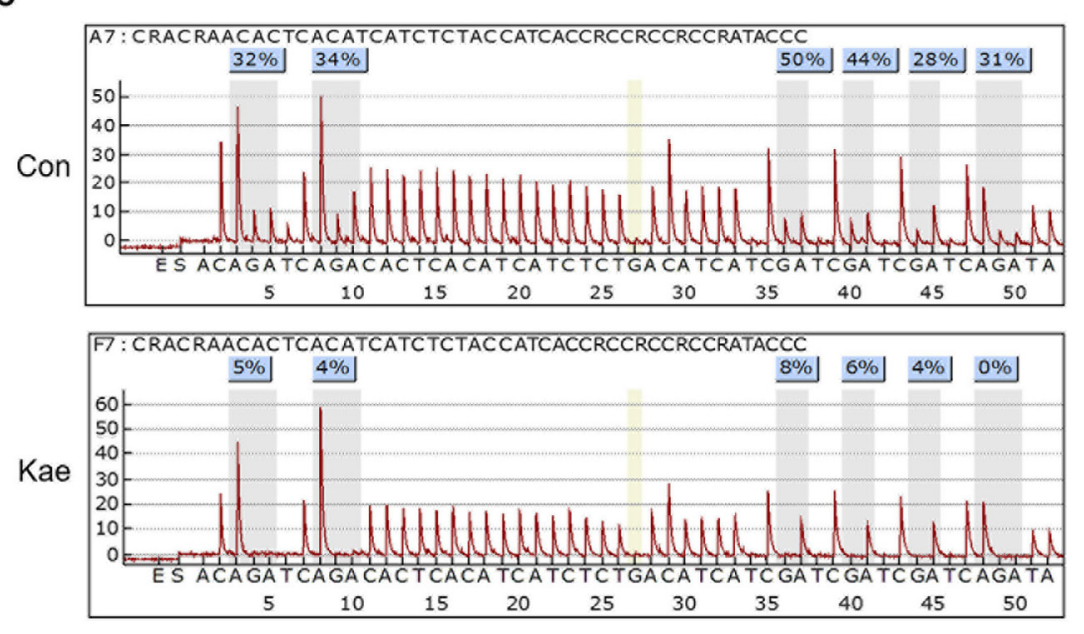

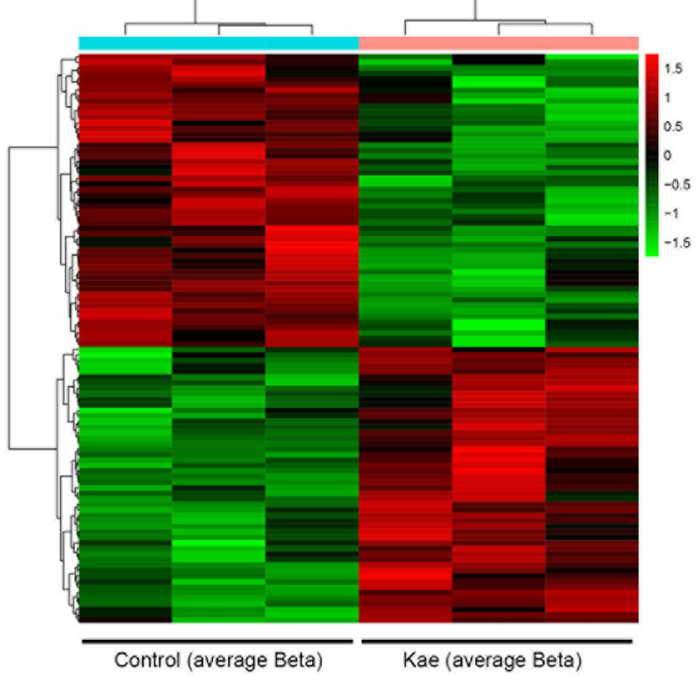


A

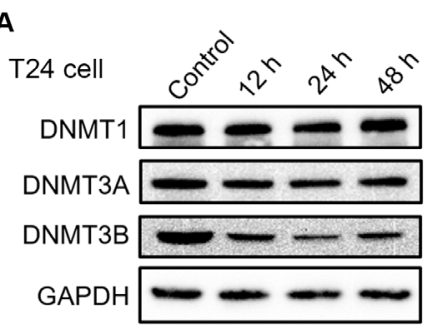

B

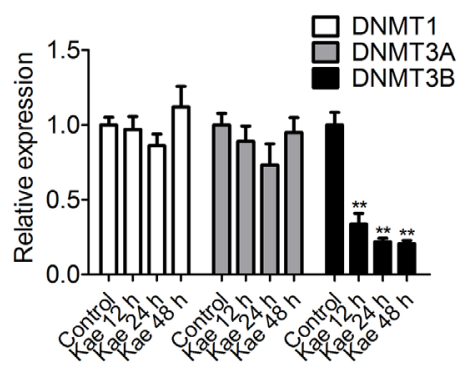

C

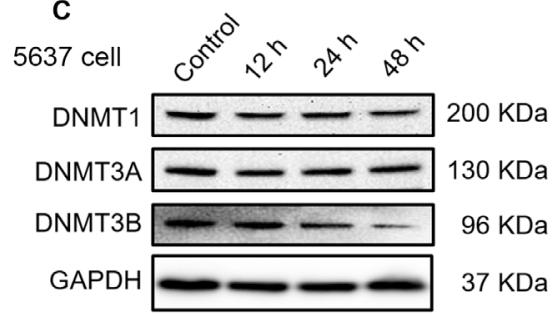

D

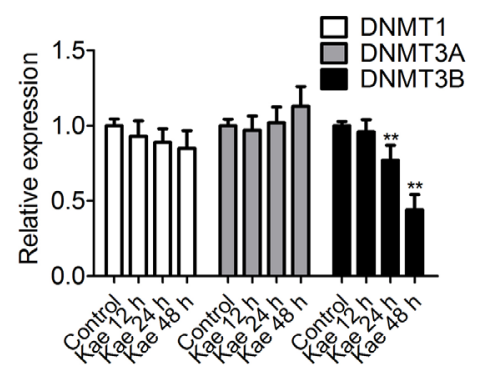

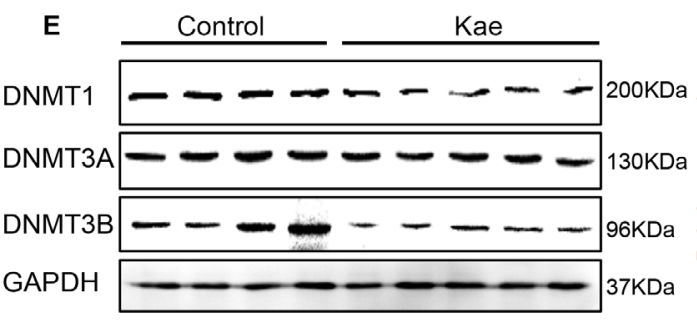

F
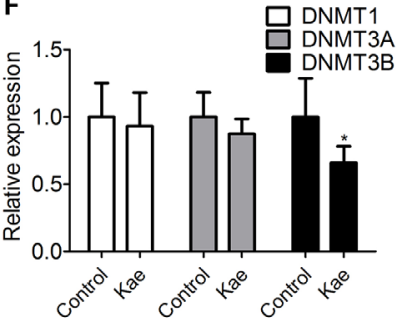

G
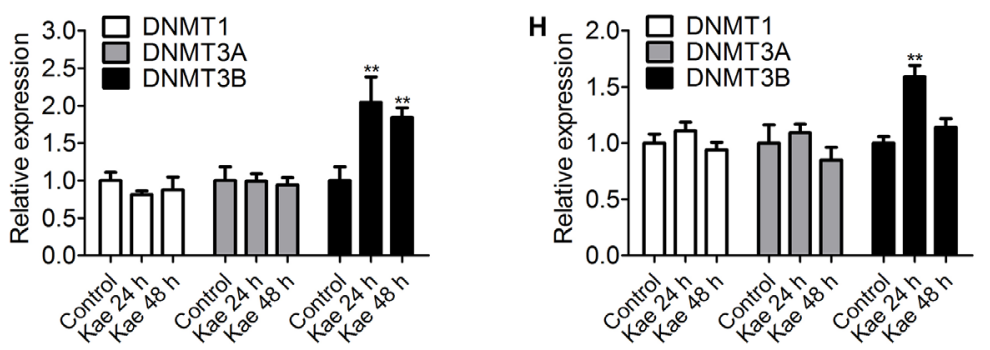

Fig. 3. Kaempferol inhibits the expression of DNMTs. (A-D) T24 and 5637 cells were treated with Kaempferol $(\mathrm{Kae}, 40 \mu \mathrm{M})$ for the indicated time. The expression of DNMT1, DNMT3A and DNMT3B was measured using western blotting (A, C). (E, F) The expression of DNMTs was detected in xenografts from nude mice bearing bladder cancer treated with Kae $(n=5)$ or vehicle $(n=4)(E)$. GAPDH was employed as an internal control. The DNMTs/GAPDH ratio for relative expression is shown (B, D, F). (G, H) The transcription of DNMTs in Kae-treated bladder cancer cells. The transcription of DNMT1, DNMT3A and DNMT3B in T24 (G) and $5637(\mathrm{H})$ cells was measured using real-time quantitative PCR. Data are expressed as the mean \pm S.D., and ${ }^{*} \mathrm{P}<0.05,{ }^{* *} \mathrm{P}<0.01$ compared with the control group.

\section{Kaempferol promotes the ubiquitination of DNMT3B}

We then investigated whether the transcription of DNMTs was regulated by Kae. T24 and 5637 cells were treated with Kae for 24 and $48 \mathrm{~h}$, respectively. The transcription of DNMT1, DNMT3A and DNMT3B was detected by using real-time qPCR. We found that Kae did not vary the transcription of DNMT1 or DNMT3A. However, the transcription of DNMT3B was extraordinarily increased induced by Kae at 24 and $48 \mathrm{~h}$ post treatment (Fig. 3G and 3H).

The amount of a protein is mostly affected by its synthesis and degradation. Because the transcription of DNMT3B is not paralleled with its protein levels after Kae treatment, we therefore speculated that Kae might activate its degradation pathway. The degradation of DNMT3B is mostly dependent on the Ub-proteasome pathway [20]. Therefore, we

\section{KARGER}


Fig. 4. Kaempferol promotes the degradation of DNMT3B through inducing ubiquitination. (A) T24 and 5637 cells were treated with or without Kaempferol (Kae; $40 \mu \mathrm{M}$ ) in the presence of cycloheximide (CHX) at $50 \mu \mathrm{g} / \mathrm{ml}$ for the indicated time, and the degradation of DNMT3B was detected using Western blotting. (B) T24 and 5637 cells were co-treated with MG132 (5 $\mu \mathrm{M}$ ) and/or Kaempferol (Kae; $40 \mu \mathrm{M}$ ) as indicated for $6 \mathrm{~h}$. The total protein in each group was immuno-precipitated. The ubiquitination status was detected in the precipitates. DNMT3B in the total protein of each group was input, and GAPDH was an internal control.

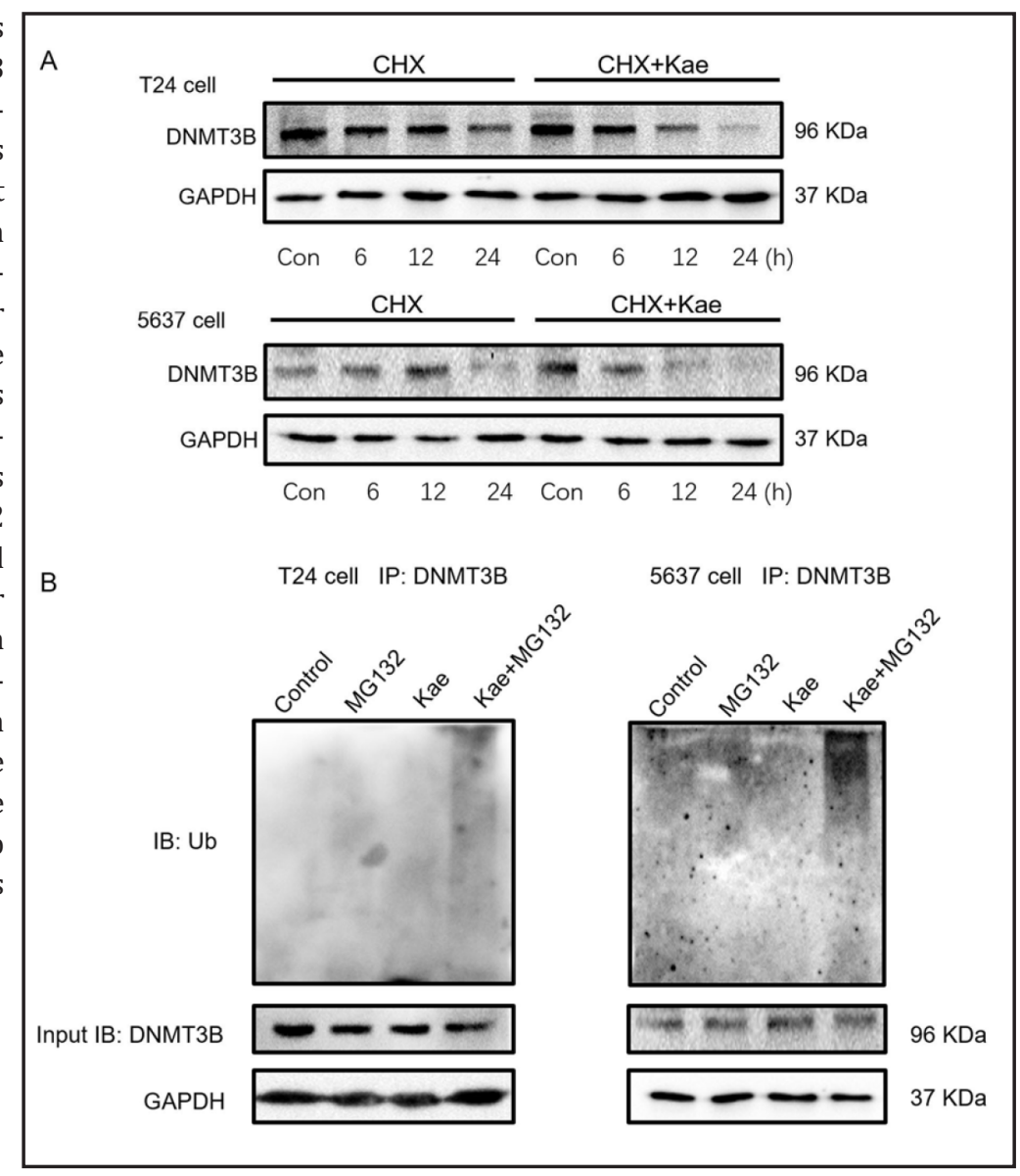

investigated the degradation of DNMT3B by using the protein synthesis inhibitor CHX. Our results indicated that the protein levels of DNMT3B were reduced within $24 \mathrm{~h}$ after CHX treatment, whereas its levels were reduced within $12 \mathrm{~h}$ in the presence of Kae (Fig. 4A). These data indicated that Kae promoted the degradation of DNMT3B in bladder cancer cells.

We next inhibited the proteasome by administering MG132, and total protein from each group was immuno-precipitated with anti-DNMT3B antibody. In the presence of MG132, we found that Kae significantly promoted the ubiquitination of DNMT3B both in T24 and 5637 cells (Fig. 4B). These results indicated that Kae downregulated the protein levels of DNMT3B by promoting its Ub-proteasome degradation.

\section{Discussion}

Targeting proteins belong to the epigenetic machinery using natural compounds is a promising area for the epigenetic therapy of cancer [21-26]. Some flavonoids, such as EGCG, can inhibit tumor growth by inhibiting DNA methylation [16]. However, the effects of Kae, a natural product that is present in many fruits and vegetables, on DNA methylation remain unclear. In this study, we found that Kae may modulate DNA methylation in bladder cancer by using epigenomic assays.

The effects of Kae on regulating histone deacetylases and non-coding RNAs have been reported $[27,28]$, which may be closely associated with bladder cancer progression [29, 30]. DNA methylation, as a common epigenetic modification, regulates the expression of genes. To our knowledge, the present study for the first time reported the effects of Kae on DNA methylation. Our data indicated that Kae did not vary the content of 5-methylcytosine, a biomarker for global DNA methylation, in bladder cancer xenografts. It modulated DNA 
methylation in specific positions. Kae induced both hyper- and hypo-methylation in the genomic DNA of bladder cancer, which may result in the differential expression of the associated genes. In this study, we found that the TSS200 region of WRN promoter was hypo-methylated and its expression was upregulated by Kae. This gene encodes a RecQ like helicase, which promotes DNA repair in response to chemotherapy drugs [31]. In addition, $R F C 3$, a replication factor that promotes tumor growth [32], was hyper-methylated in the TSS1500 region, which might induce the downregulation of its expression and inhibit tumor growth. GSTT1, encoding a glutathione S-transferase, its polymorphism was reported to be associated with the susceptibility to bladder cancer [33]. Our data indicated that Kae induced a hypo-methylation in the first exon of GSTT1, which might cause an increase of its expression. However, these results remain to be validated.

DNMTs are key enzymes in the regulation of DNA methylation. The DNA methylation is mostly relied on the expression of DNMTs. DNMT3A and DNMT3B are mostly associated with de novo DNA methylation, whereas DNMT1 is associated with maintaining the DNA methylation patterns of genes [34, 35]. The spectrum of target sequences for DNMT3A and DNMT3B are different [36], therefore, selective inactivation of DNMT3A or DNMT3B may result in different DNA methylation. In the present study, we investigated the expression of these enzymes in bladder cancer after Kae treatment. We found that Kae inhibited the expression of DNMT3B without altering the expression of DNMT1 or DNMT3A, in both T24 and 5637 cells in vitro and in the xenografts. In bladder cancer, the increasing of DNMT3B silences some tumor suppressors and thereby promote tumor progression [37]. Targeting DNMT3B may inhibit the tumor progression and increase the sensitivity to chemotherapy [38]. In Kae treated bladder cancer cells, the downregulation of DNMT3B as well as its regulated genes may contributed to the growth inhibition of bladder cancer.

The expression of DNMT3B is mostly dependent on its biosynthesis and degradation. In the present study, we observed that the transcription of DNMT1 and DNMT3A was not altered by Kae treatment. In contrast, the transcription of DNMT3B was upregulated, not paralleled with its protein levels. This effect might be a feedback to the decrease of the DNMT3B protein. In the present study, we identified that Kae promoted DNMT3B degradation in bladder cancer. Because the degradation of DNMT3B is dependent on the Ub-proteasome pathway [20], we found the Ub-conjugation of DNMT3B was indeed upregulated by Kae treatment. The activation of the Ub-proteasome pathway for the degradation of DNMT3B is dependent on the PI3K/Akt signaling pathway [20]. Phospho-Akt interacts with DNMT3B and prevents its degradation [20]. Our previous data indicated that Kae inhibits the activation of the PI3K/Akt pathway by increasing the tumor suppressor phosphatase and the tensin homolog deleted on chromosome 10 (PTEN) in bladder cancer [13]. This effect of Kae may cause the instability of DNMT3B and thereby promote its degradation via enhancing ubiquitination. Therefore, these results indicated that the downregulation of DNMT3B induced by Kae might be associated with the PI3K/Akt-associated Ub-proteasome pathway.

Taken together, our data indicate that Kae modulated DNA methylation and downregulated the level of DNMT3B through promoting its Ub-proteasome degradation. Therefore, Kae, a natural product with low toxicity, may be a novel DNMT3B inhibitor in bladder cancer.

\section{Acknowledgments}

This study was supported by grants from the Beijing Municipal Administration of Hospitals Clinical Medicine Development of Special Funding Support (No. ZYLX201604), the National Science Foundation of China (No. 81602214), the China Postdoctoral Science Foundation (2016M601065) and Beijing Municipal Science \& Technology Commission (No. Z161100000516147).

\section{KARGER}




\section{Cellular Physiology Cell Physiol Biochem 2017;41:1325-1335 \begin{tabular}{l|l} 
and Biochemistry Publisheg 10.1159/000464435 & $\begin{array}{l}\text { (c) } 2017 \text { The Author(s). Published by S. Karger AG, Basel } \\
\text { www.karger.com/cpb }\end{array}$
\end{tabular} \\ Qu et al.: Kaempferol Modulates DNA Methylation in Bladder Cancer}

\section{Disclosure Statement}

The authors declare no competing financial interests.

\section{References}

$>1$ Peres R, Furuya H, Pagano I, Shimizu Y, Hokutan K, Rosser CJ: Angiogenin contributes to bladder cancer tumorigenesis by DNMT3b-mediated MMP2 activation. Oncotarget 2016;7:43109-43123.

$\checkmark 2$ Li CW, Chen BS: Network Biomarkers of Bladder Cancer Based on a Genome-Wide Genetic and Epigenetic Network Derived from Next-Generation Sequencing Data. Dis Markers 2016;2016:4149608.

-3 Kitchen MO, Bryan RT, Emes RD, Glossop JR, Luscombe C, Cheng KK, Zeegers MP, James ND, Devall AJ, Mein CA, Gommersall L, Fryer AA, Farrell WE: Quantitative genome-wide methylation analysis of high-grade non-muscle invasive bladder cancer. Epigenetics 2016;11:237-246.

4 Palmbos PL, Wang L, Yang H, Wang Y, Leflein J, Ahmet ML, Wilkinson JE, Kumar-Sinha C, Ney GM, Tomlins SA, Daignault S, Kunju LP, Wu XR, Lotan Y, Liebert M, Ljungman ME, Simeone DM: ATDC/TRIM29 Drives Invasive Bladder Cancer Formation through miRNA-Mediated and Epigenetic Mechanisms. Cancer Res 2015;75:5155-5166.

5 Kitchen MO, Bryan RT, Haworth KE, Emes RD, Luscombe C, Gommersall L, Cheng KK, Zeegers MP, James ND, Devall AJ, Fryer AA, Farrell WE: Methylation of HOXA9 and ISL1 Predicts Patient Outcome in High-Grade Non-Invasive Bladder Cancer. PLoS One 2015;10:e0137003.

-6 Wang Y, Yu Y, Ye R, Zhang D, Li Q An D, Fang L, Lin Y, Hou Y, Xu A, Fu Y, Lu W, Chen X, Chen M, Zhang M, Jiang H, Zhang C, Dong P, Li C, Chen J, Yang G, Liu C, Cai Z, Zhou F, Wu S: An epigenetic biomarker combination of PCDH17 and POU4F2 detects bladder cancer accurately by methylation analyses of urine sediment DNA in Han Chinese. Oncotarget 2016;7:2754-2764.

-7 Maio M, Covre A, Fratta E, Di Giacomo AM, Taverna P, Natali PG, Coral S, Sigalotti L: Molecular Pathways: At the Crossroads of Cancer Epigenetics and Immunotherapy. Clin Cancer Res 2015;21:4040-4047.

-8 Azad N, Zahnow CA, Rudin CM, Baylin SB: The future of epigenetic therapy in solid tumours--lessons from the past. Nat Rev Clin Oncol 2013;10:256-266.

-9 Kim SH, Hwang KA, Choi KC: Treatment with kaempferol suppresses breast cancer cell growth caused by estrogen and triclosan in cellular and xenograft breast cancer models. J Nutr Biochem 2016;28:70-82.

-10 Lee J, Kim JH: Kaempferol Inhibits Pancreatic Cancer Cell Growth and Migration through the Blockade of EGFR-Related Pathway In Vitro. PLoS One 2016;11:e0155264.

11 Halimah E, Diantini A, Destiani DP, Pradipta IS, Sastramihardja HS, Lestari K, Subarnas A, Abdulah R, Koyama H: Induction of caspase cascade pathway by kaempferol-3-0-rhamnoside in LNCaP prostate cancer cell lines. Biomed Rep 2015;3:115-117.

$>12$ Leung HW, Lin CJ, Hour MJ, Yang WH, Wang MY, Lee HZ: Kaempferol induces apoptosis in human lung non-small carcinoma cells accompanied by an induction of antioxidant enzymes. Food Chem Toxicol 2007;45:2005-2013.

13 Xie F, Su M, Qiu W, Zhang M, Guo Z, Su B, Liu J, Li X, Zhou L: Kaempferol promotes apoptosis in human bladder cancer cells by inducing the tumor suppressor, PTEN. Int J Mol Sci 2013;14:21215-21226.

14 Dang Q Song W, Xu D, Ma Y, Li F, Zeng J, Zhu G, Wang X, Chang LS, He D, Li L: Kaempferol suppresses bladder cancer tumor growth by inhibiting cell proliferation and inducing apoptosis. Mol Carcinog 2015;54:831-840.

15 Mukherjee N, Kumar AP, Ghosh R: DNA Methylation and Flavonoids in Genitourinary Cancers. Curr Pharmacol Rep 2015;1:112-120.

16 Fang MZ, Wang Y, Ai N, Hou Z, Sun Y, Lu H, Welsh W, Yang CS: Tea polyphenol (-)-epigallocatechin-3-gallate inhibits DNA methyltransferase and reactivates methylation-silenced genes in cancer cell lines. Cancer Res 2003;63:7563-7570.

17 Qiu W, Su M, Xie F, Ai J, Ren Y, Zhang J, Guan R, He W, Gong Y, Guo Y: Tetrandrine blocks autophagic flux and induces apoptosis via energetic impairment in cancer cells. Cell Death Dis 2014;5:e1123.

18 Lu H, Fang EF, Sykora P, Kulikowicz T, Zhang Y, Becker KG, Croteau DL, Bohr VA: Senescence induced by RECQL4 dysfunction contributes to Rothmund-Thomson syndrome features in mice. Cell Death Dis 2014;5:e1226. 


\section{Cellular Physiology Cell Physiol Biochem 2017;41:1325-1335

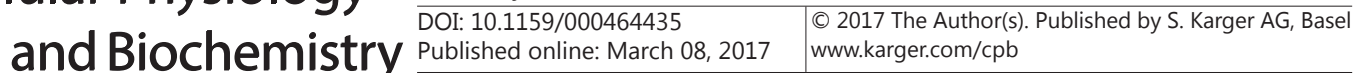

19 Nosho K, Shima K, Irahara N, Kure S, Baba Y, Kirkner GJ, Chen L, Gokhale S, Hazra A, Spiegelman D, Giovannucci EL, Jaenisch R, Fuchs CS, Ogino S: DNMT3B expression might contribute to CpG island methylator phenotype in colorectal cancer. Clin Cancer Res 2009;15:3663-3671.

20 Agarwal S, Amin KS, Jagadeesh S, Baishay G, Rao PG, Barua NC, Bhattacharya S, Banerjee PP: Mahanine restores RASSF1A expression by down-regulating DNMT1 and DNMT3B in prostate cancer cells. Mol Cancer 2013;12:99.

-21 Nandakumar V, Vaid M, Katiyar SK: (-)-Epigallocatechin-3-gallate reactivates silenced tumor suppressor genes, Cip1/p21 and p16INK4a, by reducing DNA methylation and increasing histones acetylation in human skin cancer cells. Carcinogenesis 2011;32:537-544.

22 Gu B, Ding Q Xia G, Fang Z: EGCG inhibits growth and induces apoptosis in renal cell carcinoma through TFPI-2 overexpression. Oncol Rep 2009;21:635-640.

23 Khan MA, Hussain A, Sundaram MK, Alalami U, Gunasekera D, Ramesh L, Hamza A, Quraishi U: (-)-Epigallocatechin-3-gallate reverses the expression of various tumor-suppressor genes by inhibiting DNA methyltransferases and histone deacetylases in human cervical cancer cells. Oncol Rep 2015;33:19761984.

24 Zhang Y, Wang X, Han L, Zhou Y, Sun S: Green tea polyphenol EGCG reverse cisplatin resistance of A549/ DDP cell line through candidate genes demethylation. Biomed Pharmacother 2015;69:285-290.

25 Aldawsari FS, Aguayo-Ortiz R, Kapilashrami K, Yoo J, Luo M, Medina-Franco JL, Velazquez-Martinez CA: Resveratrol-salicylate derivatives as selective DNMT3 inhibitors and anticancer agents. J Enzyme Inhib Med Chem 2016;31:695-703.

26 Paredes-Gonzalez X, Fuentes F, Su ZY, Kong AN: Apigenin reactivates Nrf2 anti-oxidative stress signaling in mouse skin epidermal JB6 P + cells through epigenetics modifications. AAPS J 2014;16:727-735.

27 Berger A, Venturelli S, Kallnischkies M, Bocker A, Busch C, Weiland T, Noor S, Leischner C, Weiss TS, Lauer UM, Bischoff SC, Bitzer M: Kaempferol, a new nutrition-derived pan-inhibitor of human histone deacetylases. J Nutr Biochem 2013;24:977-985.

28 Kim K, Kim S, Moh SH, Kang H: Kaempferol inhibits vascular smooth muscle cell migration by modulating BMP-mediated miR-21 expression. Mol Cell Biochem 2015;407:143-149.

29 Zhi Y, Pan J, Shen W, He P, Zheng J, Zhou X, Lu G, Chen Z, Zhou Z: Ginkgolide B Inhibits Human Bladder Cancer Cell Migration and Invasion Through MicroRNA-223-3p. Cell Physiol Biochem 2016;39:1787-1794.

-30 Sun DK, Wang JM, Zhang P, Wang YQ: MicroRNA-138 Regulates Metastatic Potential of Bladder Cancer Through ZEB2. Cell Physiol Biochem 2015;37:2366-2374.

31 Lee SY, Lee H, Kim ES, Park S, Lee J, Ahn B: WRN translocation from nucleolus to nucleoplasm is regulated by SIRT1 and required for DNA repair and the development of chemoresistance. Mutat Res 2015;774:4048.

-32 Shen H, Xu J, Zhao S, Shi H, Yao S, Jiang N: ShRNA-mediated silencing of the RFC3 gene suppress ovarian tumor cells proliferation. Int J Clin Exp Pathol 2015;8:8968-8975.

-33 Salinas-Sanchez AS, Sanchez-Sanchez F, Donate-Moreno MJ, Rubio-del-Campo A, Gimenez-Bachs JM, Lorenzo-Romero JG, Serrano-Oviedo L, Escribano J: Polymorphic deletions of the GSTT1 and GSTM1 genes and susceptibility to bladder cancer. BJU Int 2011;107:1825-1832.

-34 Wongtrakoongate P: Epigenetic therapy of cancer stem and progenitor cells by targeting DNA methylation machineries. World J Stem Cells 2015;7:137-148.

-35 Robert MF, Morin S, Beaulieu N, Gauthier F, Chute IC, Barsalou A, MacLeod AR: DNMT1 is required to maintain CpG methylation and aberrant gene silencing in human cancer cells. Nat Genet 2003;33:61-65.

-36 Okano M, Bell DW, Haber DA, Li E: DNA methyltransferases Dnmt3a and Dnmt3b are essential for de novo methylation and mammalian development. Cell 1999;99:247-257.

37 Li XD, Zhang JX, Jiang LJ, Wang FW, Liu LL, Liao YJ, Jin XH, Chen WH, Chen X, Guo SJ, Zhou FJ, Zeng YX, Guan XY, Liu ZW, Xie D: Overexpression of maelstrom promotes bladder urothelial carcinoma cell aggressiveness by epigenetically downregulating MTSS1 through DNMT3B. Oncogene 2016;35:6281-6292.

-38 Chen Z, Liu S, Tian L, Wu M, Ai F, Tang W, Zhao L, Ding J, Zhang L, Tang A: miR-124 and miR-506 inhibit colorectal cancer progression by targeting DNMT3B and DNMT1. Oncotarget 2015;6:38139-38150. 